\title{
Micro-Computed Tomography Applied to Museum Collections
}

\author{
Alexander D. Ball*, Richie L. Abel**, Janet Ambers***, Liesa Brierley**, Lauren E. Howard*. \\ *Electron Microscope Unit, Department of Mineralogy, The Natural History Museum, London SW7 \\ 5BD, UK \\ **Department of Surgery and Cancer, Faculty of Medicine, Imperial College London, 7th Floor East \\ Wing, Charing Cross Hospital, London W6 8RF, UK \\ ***Department of Conservation and Scientific Research, The British Museum, Great Russell Street, \\ WC1B 3DG, UK
}

In recent years the convergence between the price and resolution of micro-CT systems, the power and speed of the computers required to reconstruct the large datasets and the quality and ease of use of the commercially available reconstruction and rendering software has meant that micro-CT has now become a realistic proposition as a routine component part of a well-equipped core facility. The Natural History Museum has operated a Nikon Metrology HMX ST 225 system since 2008 and has completed more than 3,000 scans incorporating a tremendous variety of different specimen types.

Typically, skeletal material and fossils dominate research from natural history collections and this has been the basis for much of our work. However, the inventive and enquiring nature of our researchers has led to some innovative studies. For example, micro-CT was used to document and diagnose curatorial problems in our collection of Blaschka models [1]. Colleagues at the British Museum used the system to examine Neolithic artefacts [2] and we have begun to assemble a large collection of virtual specimens which can be lent or manufactured by stereolithography in lieu of loaning actual specimens. The ability to create virtual models allows very precise control over lighting and this has been used to examine flint stone tools to determine how they were knapped [3]. Virtual specimens can also be manipulated digitally - either through cross-sections, segmentation or rendering to produce detailed dissections [4] for comparison to published data. This avoids the need for destructive sampling. Alternatively, the data can be used to create movies and specimens can be reproduced using stereolithography and experimentally tested [5]. The possibilities are literally endless and the collections housed within museums become an even greater and more valuable resource as a result. References

[1] L. Brierley. Studies in conservation 54, 4 (2009) 255-267.

[2] A. Fletcher et al., Cambridge Archaeological Journal 18, 3 (2008) 309-325.

[3] S. Parfitt et al., Nature 466 (2010) 229-233.

[4] J. Dinley et al., Journal of Microscopy 238 (2010) 123-133.

[5] R.L. Abel et al., Comparative Biochemistry and Physiology A-Molecular \& Integrative Physiology Part A 155 (2010) 464-475. 


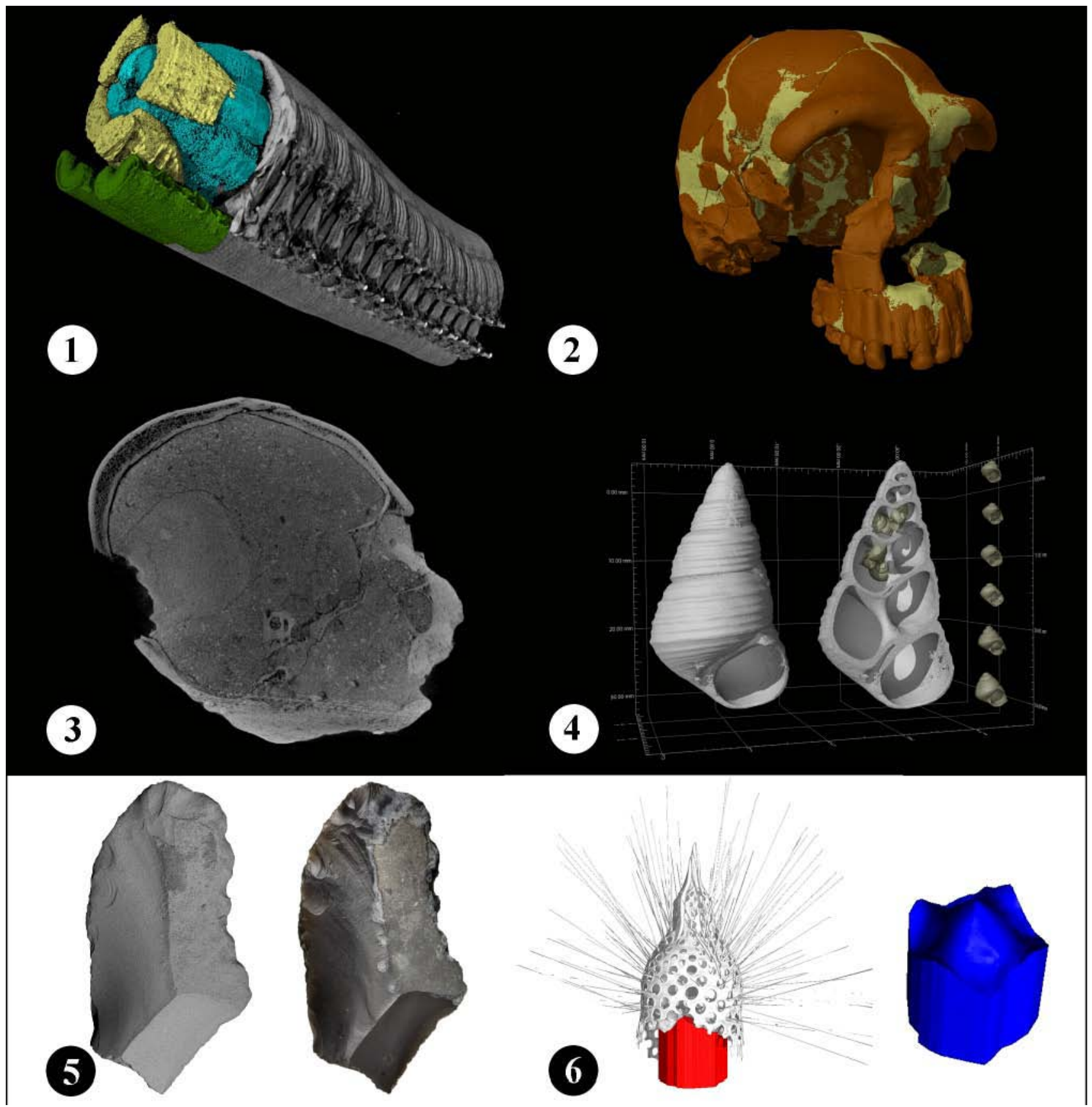

Fig. 1. Rendered reconstruction of part of the body and musculature of polychaete worm. Fig. 2. Rendered visualisation of the skull of a neanderthal (Tabun C1; NHMUK PA EM 3640), prepared to show the bone (ochre) and supporting medium (yellow) as an aid for conservation. Fig. 3. Cross-section through a decorated human skull (BM 127414) showing mud stuffing. Fig. 4. Fossil viviparous snail showing the presence of embryos within the adult. Fig. 5. CT scanned and rendered image (left) and photograph (right) of the same flint hand tool (HSB 2007 I3) showing how the control of virtual light sources in the rendered image can eliminate distracting reflections and colour variations visible in the photographic image. Fig. 6. Rendered CT scan of one of the NHM's Blaschka models, showing a stand designed to support the model (left) and the stand itself (right) ready for manufacture by stereolithography.

All images $\mathbb{C}$ The Trustees of the Natural History Museum, except (3) $\mathbb{C}$ The Trustees of the British Museum 